\title{
The Distribution of Infectious Related Symptoms in an Internet-based Syndromic Surveillance System in Rural China
}

\author{
Qi Zhao', Fuqiang Yang², Lars Palm³ ${ }^{3}$ Hui Yuan², Weirong Yan and Biao Xu*1 \\ ${ }^{1}$ School of Public Health, Fudan University, Shanghai, China; ${ }^{2}$ Jiangxi Provincial Center for Disease Control and Prevention, \\ Nanchang, China; ${ }^{3}$ Future Position X, Gavle, Sweden; ${ }^{4}$ Division of Global Health (IHCAR), Department of Public Health Sciences, \\ Karolinska Institutet, Stockholm, Sweden
}

\section{Objective}

To describe the distribution of the infectious related symptoms in an internet-based syndromic surveillance system reported by doctors in village health stations, township and county hospitals in rural Jiangxi Province, China, and to identify the major infectious diseases for syndromic surveillance in different levels of health facility.

\section{Introduction}

Syndromic surveillance system, which collects non-specific syndromes in the early stages of disease development, has great advantages in promoting early detection of epidemics and reducing the burden of disease confirmation (1). It is especially effective for surveillance in resource-poor settings, where laboratory confirmation is not possible or practical (2). Integrating syndromic surveillance with traditional case report system may generate timely, effective and sensitive information for early warning and control of infectious diseases in rural China (3). A syndromic surveillance system (ISSC) has been implemented in rural Jiangxi Province of China since August 2011.

\section{Methods}

Doctors and health workers in the healthcare surveillance units of ISSC, including village health station, township hospital and county hospital, used an internet-based electronic system to collect information of daily outpatients, which included 10 categories of infectious disease related symptoms, i.e., cough, fever, sore throat, diarrhea, headache, rash, nausea/vomit, mucocutaneous hemorrhage, convulsion and disturbance of consciousness. The data from August 1st to December 31st 2011 were extracted from database and analyzed using SPSS 16.0. The combination of symptoms was also analyzed to identify patients with the syndrome of influenza-like illness (ILI) and fever-gastrointestinal syndrome (FGS). ILI were composed by fever ( $>=38$ degree centigrade) plus cough or fever plus sore throat, and FGS were defined as fever plus vomit or diarrhea.

\section{Results}

Two county hospitals $(\mathrm{CH}), 4$ township hospitals $(\mathrm{TH})$ and 50 village health stations (VHS) were selected as surveillance unites in the pilot study during 2011/8/1 to 2011/12/31. In total, 152270 outpatient visits were reported, and 35395 patients had a chief complain of at least one surveillance symptom. Of these symptomatic patients, 24130 (68.2\%) were from VHS, 4995 (14.1\%) from TH and 6810 $(19.2 \%)$ from $\mathrm{CH}$. The proportion of patients with targeting symptom accounted for $15.5 \%, 66.4 \%$ and $23.9 \%$ of total outpatients in $\mathrm{CH}, \mathrm{TH}$ and VHS respectively. The first 3 reported symptoms were cough $(61.8 \%)$, fever $(28.4 \%)$, and sore throat $(23.4 \%)$, whereas mucocutaneous hemorrhage, convulsion and disturbance of consciousness were the least frequently reported symptoms in all surveillance units. Overall 3582 ILI and 1160 FGS cases were reported account- ing for $35 \%$ and $11 \%$ of fever cases respectively. Of the reported ILI and FGS cases, $75 \%$ ILI and $55.9 \%$ FGS cases were reported by health workers in the VHS.

\section{Conclusions}

Cough, fever and sore throat were the top surveillance symptoms, and the respiratory infectious diseases had more chance to be reported in syndromic surveillance system in rural Jiangxi Province. Training on infectious disease diagnosis especially respiratory diseases for village health workers should be enhanced since large numbers of patients are likely to visit the village health stations.

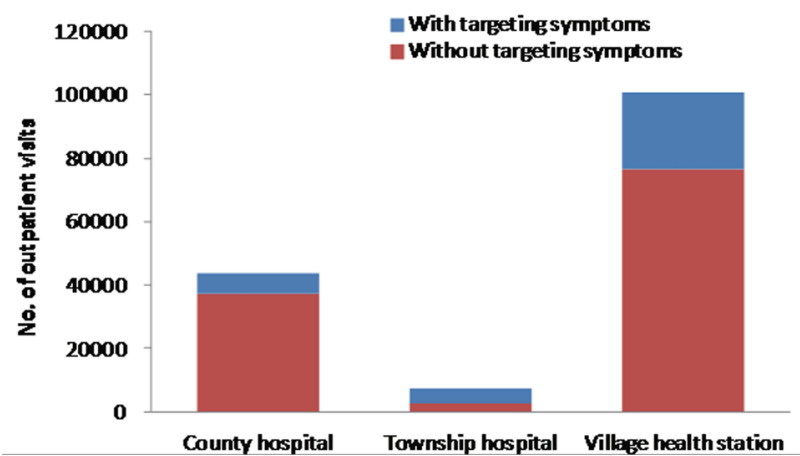

Figure1 Distributions of symptomatic patients in different level health facilities of rural China

\section{Keywords}

Syndromic surveillance; rural; influenza-likes illness; fever-gastrointestinal syndrome

\section{Acknowledgments}

This study was funded by [European Union's] [European Atomic Energy Community's] Seventh Framework Programme ([FP7/2007-2013] [FP7/2007-2011]) under grant agreement no. [241900]

\section{References}

1. Henning KJ. What is syndromic surveillance? MMWR Morb Mortal Wkly Rep 2004;53 Suppl:5-11.

2. Siswoyo H, Permana M, Larasati RP, et al. EWORS: using a syndromic-based surveillance tool for disease outbreak detection in Indonesia. BMC Proceedings 2008, 2(Suppl 3):S3

3. Reingold A. If syndromic surveillance is the answer, what is the question.Biosecur Bioterr 2003;1:1-5.

*Biao Xu

E-mail: bxu@shmu.edu.cn 